\title{
Existence of solutions for a differential inclusion problem with singular coefficients involving the $p(x)$-Laplacian
}

\author{
Guowei Dai', Ruyun Ma and Qiaozhen Ma
}

* Correspondence: daiguowei@nwnu.edu.cn Department of Mathematics, Northwest Normal University, Lanzhou 730070, P.R. China

\section{Abstract}

Using the non-smooth critical point theory we investigate the existence and multiplicity of solutions for a differential inclusion problem with singular coefficients involving the $p(x)$-Laplacian.

Mathematics Subject Classification 2000: 35D05; 35J20; 35J60; 35J70.

Keywords: $p(x)$-Laplacian, differential inclusion, singularity

\section{Introduction}

In this article, we study the existence and multiplicity of solutions for the differential inclusion problem with singular coefficients involving the $p(x)$-Laplacian of the form

$$
\left\{\begin{array}{l}
-\operatorname{div}\left(|\nabla u|^{p(x)-2} \nabla u\right) \in \lambda a_{1}(x) \partial G_{1}(x, u)+\mu a_{2}(x) \partial G_{2}(x, u) \quad \text { in } \Omega, \\
u=0 \text { on } \partial \Omega,
\end{array}\right.
$$

where the following conditions are satisfied:

(P) $\Omega$ is a bounded open domain in $\mathbb{R}^{N}, N \geq 2, p \in C(\bar{\Omega}), 1<p^{-}:=\inf _{\Omega} p(x) \leq p^{+}:=$ $\sup _{\Omega} p(x)<+\infty, \lambda, \mu \in \mathbb{R}$.

(A) For $i=1,2, a_{i} \in L^{r_{i}(x)}(\Omega), a_{i}(x)>0$ for $x \in \Omega, G_{i}(x, u)$ is measurable with respect to $x$ (for every $u \in \mathbb{R}$ ) and locally Lipschitz with respect to $u$ (for a.e. $x \in \Omega$ ), $\partial G_{i}: \Omega \times \mathbb{R} \rightarrow \mathbb{R}$ is the Clarke sub-differential of $G_{i}$ and $\left|\xi_{i}\right| \leq c_{1}+c_{2}|t|^{q_{i}(x)-1}$ for $x \in$ $\Omega, t \in \mathbb{R}$ and $\xi_{i} \in \partial G_{i}$, where $c_{i}$ is a positive constant, $r_{i}, q_{i} \in C(\bar{\Omega}), r_{i}^{-}>1, q_{i}^{-}>1, r_{i}$ $(x)>q_{i}(x)$ for all $x \in \Omega$, and

$$
q_{i}(x)<\frac{r_{i}(x)-q_{i}(x)}{r_{i}(x)} p^{*}(x), \forall x \in \bar{\Omega}
$$

here

$$
p^{*}(x)=\left\{\begin{array}{c}
\frac{N p(x)}{N-p(x)} \text { if } p(x)<N \\
\infty \text { if } p(x) \geq N .
\end{array}\right.
$$

$$
\begin{aligned}
& \left(\mathbf{A}_{1}\right) q_{1}^{+}<p^{-} . \\
& \left(\mathbf{A}_{2}\right) q_{2}^{-}>p^{+} .
\end{aligned}
$$

(c) 2012 Dai et al. ; licensee Springer. This is an open access article distributed under the terms of the Creative Commons Attribution License (http://creativecommons.org/licenses/by/2.0), which permits unrestricted use, distribution, and reproduction in any medium, provided the original work is properly cited. 
A typical example of (1.1) is the following problem involving subcritical SobolevHardy exponents of the form

$$
\left\{\begin{array}{l}
-\operatorname{div}\left(|\nabla u|^{p(x)-2} \nabla u\right) \in \lambda \frac{1}{|x|^{s_{1}(x)}} \partial G_{1}(x, u)+\mu \frac{1}{|x|^{s_{2}(x)}} \partial G_{2}(x, u) \text { in } \Omega \\
u=0 \text { on } \partial \Omega
\end{array}\right.
$$

and in this case the assumption corresponding to $(\mathbf{A})$ is the following

$(\mathrm{A})^{*} 0 \in \bar{\Omega}$, for $i=1,2, \partial G_{i}: \Omega \times \mathbb{R} \rightarrow \mathbb{R}$ is the Clarke sub-differential of $G_{i}$ and $\left|\xi_{i}\right| \leq c_{1}+c_{2}|t|^{q_{i}(x)-1}$ for $x \in \Omega, t \in \mathbb{R}$ and $\xi_{i} \in \partial G_{i}$, where $c_{i}$ is a positive constant, $s_{i}, q_{i} \in C(\bar{\Omega}), 0 \leq s_{i}^{-} \leq s_{i}^{+}<N, q_{i}^{-}>1$, and

$$
q_{i}(x)<\frac{N-s_{i}(x) q_{i}(x)}{N} p^{*}(x), \forall x \in \bar{\Omega} .
$$

The operator $-\operatorname{div}\left(|\nabla u|^{p(x)-2} \nabla u\right)$ is said to be the $p(x)$-Laplacian, and becomes $p$-Laplacian when $p(x) \equiv p$ (a constant). The $p(x)$-Laplacian possesses more complicated nonlinearities than the $p$-Laplacian; for example, it is inhomogeneous. The study of various mathematical problems with variable exponent growth condition has been received considerable attention in recent years. These problems are interesting in applications and raise many difficult mathematical problems. One of the most studied models leading to problem of this type is the model of motion of electro-rheological fluids, which are characterized by their ability to drastically change the mechanical properties under the influence of an exterior electro-magnetic field [1,2]. Problems with variable exponent growth conditions also appear in the mathematical modeling of stationary thermo-rheological viscous flows of non-Newtonian fluids and in the mathematical description of the processes filtration of an ideal baro-tropic gas through a porous medium [3,4]. Another field of application of equations with variable exponent growth conditions is image processing [5]. The variable nonlinearity is used to outline the borders of the true image and to eliminate possible noise. We refer the reader to [6-11] for an overview of and references on this subject, and to [12-21] for the study of the $p(x)$-Laplacian equations and the corresponding variational problems.

Since many free boundary problems and obstacle problems may be reduced to partial differential equations with discontinuous nonlinearities, the existence of multiple solutions for Dirichlet boundary value problems with discontinuous nonlinearities has been widely investigated in recent years. Chang [22] extended the variational methods to a class of non-differentiable functionals, and directly applied the variational methods for non-differentiable functionals to prove some existence theorems for PDE with discontinuous nonlinearities. Later Kourogenis and Papageorgiou [23] obtained some nonsmooth critical point theories and applied these to nonlinear elliptic equations at resonance, involving the $p$-Laplacian with discontinuous nonlinearities. In the celebrated work [24,25], Ricceri elaborated a Ricceri-type variational principle and a three critical points theorem for the Gâteaux differentiable functional, respectively. Later, Marano and Motreanu [26,27] extended Ricceri's results to a large class of non-differentiable functionals and gave some applications to differential inclusion problems involving the $p$-Laplacian with discontinuous nonlinearities. 
In [21], by means of the critical point theory, Fan obtain the existence and multiplicity of solutions for (1.1) under the condition of $G_{i}(x, \cdot) \in C^{1}(\mathbb{R})$ and $g_{i}=G_{i}^{\prime}$ satisfying the Carathéodory condition for $i=1,2, x \in \Omega$. The aim of the present article is to generalize the main results of [21] to the case of the functional of problem (1.1) is nonsmooth.

This article is organized as follows: In Section 2, we present some necessary preliminary knowledge on variable exponent Sobolev spaces and the generalized gradient of the locally Lipschitz function; In Section 3, we give the variational principle which is needed in the sequel; In Section 4, using the critical point theory, we prove the existence and multiplicity results for problem (1.1).

\section{Preliminaries}

\subsection{Variable exponent Sobolev spaces}

Let $\Omega$ be a bounded open subset of $\mathbb{R}^{N}$, denote $L_{+}^{\infty}(\Omega)=\left\{p \in L^{\infty}(\Omega): \operatorname{essinf}_{\Omega} p(x) \geq 1\right\}$.

For $p \in L_{+}^{\infty}(\Omega)$, denote

$$
p^{-}=p^{-}(\Omega)=\text { ess } \inf _{x \in \Omega} p(x), \quad p^{+}=p^{+}(\Omega)=\text { ess } \sup _{x \in \Omega} p(x) .
$$

On the basic properties of the space $W^{1, p(x)}(\Omega)$ we refer to [7,28-30]. Here we display some facts which will be used later.

Denote by $\mathbf{S}(\Omega)$ the set of all measurable real functions defined on $\Omega$. Two functions in $\mathbf{S}(\Omega)$ are considered as the same element of $\mathbf{S}(\Omega)$ when they are equal almost everywhere. For $p \in L_{+}^{\infty}(\Omega)$, define the spaces $L^{p(x)}(\Omega)$ and $W^{1, p(x)}(\Omega)$ by

$$
L^{p(x)}(\Omega)=\left\{u \in \mathrm{S}(\Omega): \int_{\Omega}|u(x)|^{p(x)} d x<\infty\right\}
$$

with the norm

$$
|u|_{L^{p(x)}(\Omega)}=|u|_{p(x)}=\inf \left\{\lambda>0: \int_{\Omega}\left|\frac{u(x)}{\lambda}\right|^{p(x)} d x \leq 1\right\},
$$

and

$$
W^{1, p(x)}(\Omega)=\left\{u \in L^{p(x)}(\Omega):|\nabla u| \in L^{p(x)}(\Omega)\right\}
$$

with the norm

$$
\|\left. u\right|_{W^{1, p(x)}(\Omega)}=|u|_{L^{p(x)}(\Omega)}+|\nabla u|_{L^{p(x)}(\Omega)} .
$$

Denote by $W_{0}^{1, p(x)}(\Omega)$ the closure of $C_{0}^{\infty}(\Omega)$ in $W^{1, p(x)}(\Omega)$. Hereafter, we always assume that $p^{-}>1$.

Proposition 2.1. $[7,31]$ The spaces $L^{p(x)}(\Omega), W^{1, p(x)}(\Omega)$ and $W_{0}^{1, p(x)}(\Omega)$ are separable and reflexive Banach spaces.

Proposition 2.2. [7,31] The conjugate space of $L^{p(x)}(\Omega)$ is $L^{p^{0}(x)}(\Omega)$, where $\frac{1}{p(x)}+\frac{1}{p^{0}(x)}=1$. For any $u \in L^{p(x)}(\Omega)$ and $v \in L^{p^{0}(x)}(\Omega), \int_{\Omega}|u v| d x \leq 2|u|_{p(x)}|v|_{p^{0}(x)}$. 
Proposition 2.3. $[7,31] \operatorname{In} W_{0}^{1, p(x)}(\Omega)$ the Poincaré inequality holds, that is, there exists a positive constant $c$ such that

$$
|u|_{L^{p(x)}(\Omega)} \leq c|\nabla u|_{L^{p(x)}(\Omega)}, \forall u \in W_{0}^{1, p(x)}(\Omega) .
$$

So $|\nabla u|_{L^{p(x)}(\Omega)}$ is an equivalent norm in $W_{0}^{1, p(x)}(\Omega)$.

Proposition 2.4. $[7,28,29,31]$ Assume that the boundary of $\Omega$ possesses the cone property and $p \in C(\bar{\Omega})$. If $q \in C(\bar{\Omega})$ and $1 \leq q(x)<p^{*}(x)$ for $x \in \bar{\Omega}$, then there is a compact embedding $W^{1, p(x)}(\Omega) \rightarrow L^{q(x)}(\Omega)$.

Let us now consider the weighted variable exponent Lebesgue space.

Let $a \in \mathbf{S}(\Omega)$ and $a(x)>0$ for $x \in \Omega$. Define

$$
L_{a(x)}^{p(x)}(\Omega)=\left\{u \in \mathbf{S}(\Omega): \int_{\Omega} a(x)|u(x)|^{p(x)} d x<\infty\right\}
$$

with the norm

$$
|u|_{L_{a(x)}^{p(x)}(\Omega)}=|u|_{(p(x), a(x))}=\inf \left\{\lambda>0: \int_{\Omega} a(x)\left|\frac{u(x)}{\lambda}\right|^{p(x)} d x \leq 1\right\},
$$

then $L_{a(x)}^{p(x)}(\Omega)$ is a Banach space. The following proposition follows easily from the definition of $|u|_{L_{a(x)}^{p(x)}(\Omega)}$.

Proposition 2.5. (see [7,31]) Set $\rho(u)=\int_{\Omega} a(x)|u(x)|^{p(x)} \mathrm{dx}$. For $u, u_{k} \in L_{a(x)}^{p(x)}(\Omega)$, we have

(1) For $u \neq 0,|u|_{(p(x), a(x))}=\lambda \Leftrightarrow \rho\left(\frac{u}{\lambda}\right)=1$.

(2) $|u|_{(p(x), a(x))}<1(=1 ;>1) \Leftrightarrow \rho(u)<1(=1 ;>1)$.

(3) If $|u|_{(p(x), a(x))}>1$, then $|u|_{(p(x), a(x))}^{p^{-}} \leq \rho(u) \leq|u|_{(p(x), a(x))}^{p^{+}}$.

(4) If $|u|_{(p(x), a(x))}<1$, then $|u|_{(p(x), a(x)}^{p^{+}} \leq \rho(u) \leq|u|_{(p(x), a(x))}^{p^{-}}$.

(5) $\lim _{k \rightarrow \infty}\left|u_{k}\right|_{(p(x), a(x))}=0 \Leftrightarrow \lim _{k \rightarrow \infty} \rho\left(u_{k}\right)=0$.

(6) $\left|u_{k}\right|_{(p(x), a(x))} \rightarrow \infty \Leftrightarrow \rho\left(u_{k}\right) \rightarrow \infty$.

Proposition 2.6. (see [21]) Assume that the boundary of $\Omega$ possesses the cone property and $p \in C(\bar{\Omega})$. Suppose that $a \in L^{r}\left({ }^{x}\right)(\Omega), a(x)>0$ for $x \in \Omega, r \in C(\bar{\Omega})$ and $r^{-}>1$. If $q \in C(\bar{\Omega})$ and

$$
1 \leq q(x)<\frac{r(x)-1}{r(x)} p^{*}(x):=p_{a(x)}^{*}(x), \forall x \in \bar{\Omega},
$$

then there is a compact embedding $W^{1, p(x)}(\Omega) \rightarrow L_{a(x)}^{q(x)}(\Omega)$.

The following proposition plays an important role in the present article. 
Proposition 2.7. Assume that the boundary of $\Omega$ possesses the cone property and $p \in C(\bar{\Omega})$. Suppose that $a \in L^{r}\left({ }^{x}\right)(\Omega), a(x)>0$ for $x \in \Omega, r \in C(\bar{\Omega})$ and $r(x)>q(x)$ for all $x \in \Omega$. If $q \in C(\bar{\Omega})$ and

$$
1 \leq q(x)<\frac{r(x)-q(x)}{r(x)} p^{*}(x), \forall x \in \bar{\Omega},
$$

then there is a compact embedding $W^{1, p(x)}(\Omega) \rightarrow L_{(a(x))^{q(x)}}^{q(x)}(\Omega)$.

Proof. Set $r_{1}(x)=\frac{r(x)}{q(x)}$, then $r_{1}^{-}>1$ and $(a(x))^{q(x)} \in L^{r_{1}(x)}(\Omega)$. Moreover, from (2.2) we can get

$$
1 \leq q(x)<\frac{r_{1}(x)-1}{r_{1}(x)} p^{*}(x), \forall x \in \bar{\Omega} .
$$

Using Proposition 2.6, we see that the embedding $W^{1, p(x)}(\Omega) \rightarrow L_{(a(x))^{q(x)}}^{q(x)}(\Omega)$ is compact.

\subsection{Generalized gradient of the locally Lipschitz function}

Let $(X,\|\cdot\|)$ be a real Banach space and $X^{*}$ be its topological dual. A function $f: X \rightarrow$ $\mathbb{R}$ is called locally Lipschitz if each point $u \in X$ possesses a neighborhood $\Omega_{u}$ such that $\left|f\left(u_{1}\right)-f\left(u_{2}\right)\right| \leq L|| u_{1}-u_{2}||$ for all $u_{1}, u_{2} \in \Omega_{u}$, for a constant $L>0$ depending on $\Omega_{u}$. The generalized directional derivative of $f$ at the point $u \in X$ in the direction $v \in X$ is

$$
f^{0}(u, v)=\limsup _{w \rightarrow u, t \rightarrow 0} \frac{1}{t}(f(w+t v)-f(w)) .
$$

The generalized gradient of $f$ at $u \in X$ is defined by

$$
\partial f(u)=\left\{u^{*} \in X^{*}:\left\langle u^{*}, \varphi\right\rangle \leq f^{0}(u ; \varphi) \text { for all } \varphi \in X\right\},
$$

which is a non-empty, convex and $w^{*}$-compact subset of $X$, where $\langle\cdot, \cdot\rangle$ is the duality pairing between $X^{*}$ and $X$. We say that $u \in X$ is a critical point of $f$ if $0 \in \partial f(u)$. For further details, we refer the reader to Chang [22].

We list some fundamental properties of the generalized directional derivative and gradient that will be used throughout the article.

Proposition 2.8. (see [22,32]) (1) Let $j: X \rightarrow \mathbb{R}$ be a continuously differentiable function. Then $\partial j(u)=\left\{j^{\prime}(u)\right\}, j^{0}(u ; z)$ coincides with $\left\langle j^{\prime}(u), z\right\rangle_{X}$ and $(f+j)^{0}(u, z)=f^{0}(u ; z)+$ $\left\langle j^{\prime}(u), z\right\rangle_{X}$ for all $u, z \in X$.

(2) The set-valued mapping $u \rightarrow \partial f(u)$ is upper semi-continuous in the sense that for each $u_{0} \in X, \varepsilon>0, v \in X$, there is a $\delta>0$, such that for each $w \in \partial f(u)$ with $\left\|w-u_{0}\right\|$ $<\delta$, there is $w_{0} \in \partial f\left(u_{0}\right)$

$$
\left|\left\langle w-w_{0}, v\right\rangle\right|<\varepsilon .
$$

(3) (Lebourg's mean value theorem) Let $u$ and $v$ be two points in $X$. Then there exists a point $w$ in the open segment joining $u$ and $v$ and $x_{w}^{*} \in \partial f(w)$ such that 


$$
f(u)-f(v)=\left\langle x_{w^{\prime}}^{*} u-v\right\rangle_{X} .
$$

(4) The function

$$
m(u)=\min _{w \in \partial f(u)} w_{X^{*}}
$$

exists, and is lower semi continuous; i.e., $\liminf _{u \rightarrow u_{0}} m(u) \geq m\left(u_{0}\right)$.

In the following we need the nonsmooth version of Palais-Smale condition.

Definition 2.1. We say that $\phi$ satisfies the (PS) $)_{c}$-condition if any sequence $\left\{u_{n}\right\} \subset X$ such that $\phi\left(u_{n}\right) \rightarrow c$ and $m\left(u_{n}\right) \rightarrow 0$, as $n \rightarrow+\infty$, has a strongly convergent subsequence, where $m\left(u_{n}\right)=\inf \left\{\left\|u^{*}\right\|_{X^{*}}: u^{*} \in \partial \phi\left(u_{n}\right)\right\}$.

In what follows we write the (PS) $)_{c}$-condition as simply the PS-condition if it holds for every level $c \in \mathbb{R}$ for the Palais-Smale condition at level $c$.

\section{Variational principle}

In this section we assume that $\Omega$ and $p(x)$ satisfy the assumption (P). For simplicity we write $X=W_{0}^{1, p(x)}(\Omega)$ and $\|u\|=|\nabla u|_{p}\left(x_{x}\right)$ for $u \in X$. Denote by $u_{n} \rightarrow u$ and $u_{n} \rightarrow u$ the weak convergence and strong convergence of sequence $\left\{u_{n}\right\}$ in $X$, respectively, denote by $c$ and $c_{i}$ the generic positive constants, $B_{\rho}=\{u \in X:\|u\|<\rho\}, S_{\rho}=\{u \in X:\|u\|=\rho\}$.

Set

$$
F(x, t)=\lambda a_{1}(x) G_{1}(x, t)+\mu a_{2}(x) G_{2}(x, t),
$$

where $a_{i}$ and $G_{i}(i=1,2)$ are as in $(\mathbf{A})$.

Define the integral functional

$$
\varphi(u)=\int_{\Omega} \frac{1}{p(x)}|\nabla u|^{p(x)} d x-\int_{\Omega} F(x, u) d x, \forall u \in X .
$$

We write

$$
J(u)=\int_{\Omega} \frac{1}{p(x)}|\nabla u|^{p(x)} d x, \Psi(u)=\int_{\Omega} F(x, u) d x,
$$

then it is easy to see that $J \in C^{1}(X, \mathbb{R})$ and $\phi=J-\Psi$.

Below we give several propositions that will be used later.

Proposition 3.1. (see [19]) The functional $J: X \rightarrow \mathbb{R}$ is convex. The mapping $\rho: X \rightarrow$ $X^{*}$ is a strictly monotone, bounded homeomorphism, and is of $\left(S_{+}\right)$type, namely

$$
u_{n} \rightarrow u \text { and } \varlimsup_{n \rightarrow \infty} J /\left(u_{n}\right)\left(u_{n}-u\right) \leq 0 \text { implies } u_{n} \rightarrow u \text {. }
$$

Proposition 3.2. $\Psi$ is weakly-strongly continuous, i.e., $u_{n} \rightarrow u$ implies $\Psi\left(u_{n}\right) \rightarrow \Psi(u)$.

Proof. Define $\Upsilon_{1}=\int_{\Omega} G_{1}(x, u) d x$ and $\Upsilon_{2}=\int_{\Omega} G_{2}(x, u) d x$. In order to prove $\Psi$ is weakly-strongly continuous, we only need to prove $\Upsilon_{1}$ and $\Upsilon_{2}$ are weakly-strongly continuous. Since the proofs of $\Upsilon_{1}$ and $\Upsilon_{2}$ are identical, we will just prove $\Upsilon_{1}$.

We assume $u_{n} \rightarrow u$ in $X$. Then by Proposition 2.8.3, we have

$$
\begin{aligned}
\Upsilon_{1}\left(u_{n}\right)-\Upsilon_{1}(u) & =\int_{\Omega}\left(G_{1}\left(x, u_{n}\right)-G_{1}(x, u)\right) d x \\
& =\int_{\Omega} \xi_{n}(x)\left(u_{n}-u\right) d x
\end{aligned}
$$


where $\xi_{n} \in \partial G_{1}\left(, \tau_{n}(x)\right)$ for some $\tau_{n}(x)$ in the open segment joining $u$ and $u_{n}$. From Chang [22] we know that $\xi_{n} \in L^{q_{1}^{0}(x)}(\Omega)$. So using Proposition 2.5, we have

$$
\Upsilon_{1}\left(u_{n}\right)-\Upsilon_{1}(u) \rightarrow 0 .
$$

Proposition 3.3. Assume (A) holds and $F$ satisfies the following condition:

(B) $F(x, u) \leq \theta \lambda a_{1}(x)\left\langle\xi_{1}, u\right\rangle+\theta \mu a_{2}(x)\left\langle\xi_{2}, u\right\rangle+b(x)+\sum_{i=1}^{m} d_{i}(x)|u|^{k_{i}(x)}$ for a.e. $x \in \Omega$, all $u \in X$ and $\xi_{1} \in \partial G_{1}, \xi_{2} \in \partial G_{2}$, where $\theta$ is a constant, $h_{i}, k_{i} \in C(\bar{\Omega}), k_{i}(x)<\frac{h_{i}(x)-1}{h_{i}(x)} p^{*}(x)$ for $x \in \bar{\Omega}, k_{i}^{+}<p^{-}$, $h_{i}, k_{i} \in C(\bar{\Omega}), k_{i}(x)<\frac{h_{i}(x)-1}{h_{i}(x)} p^{*}(x)$ for $x \in \bar{\Omega}, k_{i}^{+}<p^{-}$.

Then $\phi$ satisfies the nonsmooth (PS) condition on $X$.

Proof. Let $\left\{u_{n}\right\}$ be a nonsmooth (PS) sequence, then by $(B)$ we have

$$
\begin{aligned}
c+1+\left\|u_{n}\right\| & \geq \varphi\left(u_{n}\right)-\theta\left\langle\omega, u_{n}\right\rangle \\
& =\int_{\Omega}\left(\frac{1}{p(x)}-\theta\right)\left|\nabla u_{n}\right|^{p(x)} d x \\
& -\int_{\Omega}\left(F\left(x, u_{n}\right)-\theta \lambda a_{1}(x)\left\langle\xi_{1}, u_{n}\right\rangle-\theta \mu a_{2}(x)\left\langle\xi_{2}, u_{n}\right\rangle\right) d x \\
& \geq\left(\frac{1}{p^{+}}-\theta\right)\left\|u_{n}\right\|^{p^{-}}-c_{1}-\int_{\Omega}\left(b(x)+\sum_{i=1}^{m} d_{i}(x)\left|u_{n}\right|^{k_{i}(x)}\right) d x \\
& \geq\left(\frac{1}{p^{+}}-\theta\right)\left\|u_{n}\right\|^{p^{-}}-c_{2}-\sum_{i=1}^{m}\left|u_{n}\right|_{\left(k_{i}(x), d_{i}(x)\right)}^{k^{+}} \\
& \geq\left(\frac{1}{p^{+}}-\theta\right)\left\|u_{n}\right\|^{p^{-}}-c_{2}-c_{3} \sum_{i=1}^{m}|| u_{n} \|^{k_{i}^{+}},
\end{aligned}
$$

and consequently $\left\{u_{n}\right\}$ is bounded.

Thus by passing to a subsequence if necessary, we may assume that $u_{n} \rightarrow u$ in $X$ as $n \rightarrow \infty$. We have

$$
\left\langle J^{\prime}\left(u_{n}\right), u_{n}-u\right\rangle-\int_{\Omega} \lambda \xi_{1 n}(x) a_{1}(x)\left(u_{n}-u\right)-\int_{\Omega} \mu \xi_{2 n}(x) a_{2}(x)\left(u_{n}-u\right) d x \leq \varepsilon_{n}\left\|u_{n}-u\right\|
$$

with $\varepsilon_{n} \downarrow 0$, where $\xi_{i n}(x) \in \partial G_{i}\left(x, u_{n}\right)$ for a.e. $x \in \Omega, i=1$, 2. From Chang [22] or Theorem 1.3 .10 of [33], we know that $\xi_{\text {in }}(x) \in L^{q_{1}^{0}(x)}, i=1,2$. Since $X$ is embedded compactly in $L_{\left(a_{i}(x)\right)^{q_{i}(x)}}^{q_{i}(x)}(\Omega)$, we have that $u_{n} \rightarrow u$ as $n \rightarrow \infty$ in $L_{\left(a_{i}(x)\right)^{q_{i}(x)}}^{q_{(}(x)}(\Omega), i=1,2$. So using Proposition 2.2, we have

$$
\int_{\Omega} \xi_{\text {in }}(x) a_{i}(x)\left(u_{n}-u\right) d x \rightarrow 0 \text { as } n \rightarrow \infty \quad, i=1,2 .
$$

Therefore we obtain $\limsup _{n \rightarrow \infty}\left\langle J \prime\left(u_{n}\right), u_{n}-u\right\rangle \leq 0$. But we know that $\rho$ is a mapping of type $\left(S_{+}\right)$. Thus we have 


$$
u_{n} \rightarrow u \text { in } X
$$

Remark 3.1. Note that our condition (1.2) is stronger than (1.2) of [21]. Because $\Psi^{\prime}$ is weakly-strongly continuous in [21], to verify that $\phi$ satisfies (PS) condition on $X$, it is enough to verify that any (PS) sequence is bounded. However, in this paper we do not know whether $\xi(u)$ is weakly-strongly continuous, where $\xi(u) \in \rightarrow \Psi$. Therefore, it will be very useful to consider this problem.

Below we denote

$$
F_{1}(x, t)=\lambda a_{1}(x) G_{1}(x, t), F_{2}(x, t)=\mu a_{2}(x) G_{2}(x, t) .
$$

We shall use the following conditions.

$\left(\mathbf{B}_{1}\right) \exists c_{0}>0$ such that $G_{2}(x, t) \geq-c_{0}$ for $x \in \Omega$ and $t \in \mathbb{R}$.

$\left(\mathbf{B}_{2}\right) \exists \theta \in\left(0, \frac{1}{p^{+}}\right)$and $M>0$ such that $0<G_{2}(x, u) \leq \theta\left\langle u, \xi_{2}\right\rangle$ for $x \in \Omega, u \in X$ and $|u| \geq M, \xi_{2} \in \rightarrow G_{2}$.

Corollary 3.1. Assume (P), (A) and ( $\left.\mathbf{A}_{1}\right)$ hold. Then $\phi$ satisfies nonsmooth (PS) condition on $X$ provided either one of the following conditions is satisfied.

(1). $\lambda \in \mathbb{R}$ and $\mu=0$.

(2). $\lambda \in \mathbb{R}, \mu=0$ and $\left(\mathbf{B}_{1}\right)$ holds.

(3). $\lambda \in \mathbb{R}, \mu \in \mathbb{R}$ and $\left(\mathbf{B}_{2}\right)$ holds.

Proof. In case (1) or (2), we have, for $x \in \Omega$ and $t \in \mathbb{R}$,

$$
F(x, t) \leq F_{1}(x, t)+|\mu| c_{0} a_{2}(x) \leq\left(c_{1} a_{1}(x)+|\mu| c_{0} a_{2}(x)\right)+c_{2} a_{1}(x)|t|^{q_{1}(x)},
$$

which shows that the condition (B) with $\theta=0$ is satisfied.

In case (3), noting that $\left(\mathbf{B}_{2}\right)$ and (A) imply $\left(\mathbf{B}_{1}\right)$, by the conclusion (1) and (2) we know $\phi$ satisfies (PS) condition if $\mu \leq 0$. Below assume $\mu>0$. The conditions $\left(\mathbf{B}_{2}\right)$ and (A) imply that, for $x \in \Omega$ and $u \in X$,

$$
G_{2}(x, u) \leq \theta\left\langle u, \xi_{2}\right\rangle+c_{3}, \text { and } F_{2}(x, u) \leq \theta \mu \mathbf{a}_{2}(x)\left\langle u, \xi_{2}\right\rangle+c_{3} \mu a_{2}(x),
$$

so we have

$$
\begin{aligned}
F(x, u)-\theta \lambda a_{1}(x)\left\langle\xi_{1}, u\right\rangle-\theta \mu a_{2}(x)\left\langle\xi_{2}, u\right\rangle & =\left(F_{1}(x, u)-\theta \lambda a_{1}(x)\left\langle\xi_{1}, u\right\rangle\right) \\
& +\left(F_{2}(x, u)-\theta \mu a_{2}(x)\left\langle\xi_{2}, u\right\rangle\right) \\
& \leq c_{1} a_{1}(x)+c_{2} a_{1}(x)|u|^{q_{1}(x)}+c_{3} \mu a_{2}(x)
\end{aligned}
$$

which shows (B) holds. The proof is complete.

As $X$ is a separable and reflexive Banach space, there exist (see [[34], Section 17]) $\left\{e_{n}\right\}_{n=1}^{\infty} \subset X$ and $\left\{f_{n}\right\}_{n=1}^{\infty} \subset X^{*}$ such that

$$
\begin{aligned}
& f_{n}\left(e_{m}\right)=\delta_{n, m}= \begin{cases}1 & \text { if } n=m \\
0 & \text { if } n \neq m,\end{cases} \\
& X=\overline{\operatorname{span}}\left\{e_{n}: n=1,2, \ldots,\right\}, X^{*}=\overline{\operatorname{span}}^{W *}\left\{f_{n}: n=1,2, \ldots,\right\} .
\end{aligned}
$$


For $k=1,2, \ldots$, denote

$$
X_{k}=\operatorname{span}\left\{e_{k}\right\}, Y_{k}=\oplus_{j=1}^{k} X_{j}, Z_{k}=\overline{\oplus_{j=k}^{\infty} X_{j}} .
$$

Proposition 3.5. [35] Assume that $\Psi: X \rightarrow \mathbb{R}$ is weakly-strongly continuous and $\Psi(0)$ $=0$. Let $\gamma>0$ be given. Set

$$
\beta_{k}=\beta_{k}(\gamma)=\sup _{u \in Z_{k},\|u\| \leq \gamma}|\Psi(u)|
$$

Then $\beta_{k} \rightarrow 0$ as $k \rightarrow \infty$.

Proposition 3.6. (Nonsmooth Mountain pass theorem, see [23,33]) If $X$ is a reflexive Banach space, $\phi: X \rightarrow \mathbb{R}$ is a locally Lipschitz function which satisfies the nonsmooth

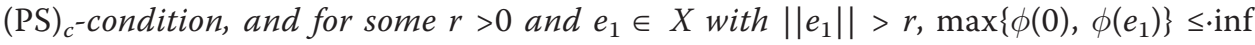
$\{\phi(u):\|u\|=r\}$. Then $\phi$ has a nontrivial critical $u \in X$ such that the critical value $c=$ $\phi(u)$ is characterized by the following minimax principle

$$
c=\inf _{\gamma \in \Gamma} \max _{t \in[0,1]} \varphi(\gamma(t)
$$

where $\Gamma=\left\{\gamma \in C([0,1], X): \gamma(0)=0, \gamma(1)=e_{1}\right\}$.

Proposition 3.7. (Nonsmooth Fountain theorem, see [36]) Assume ( $\left.F_{1}\right) X$ is a Banach space, $\phi: X \rightarrow \mathbb{R}$ be an invariant locally Lipschitz functional, the subspaces $X_{k}$, $Y_{k}$ and $Z_{k}$ are defined by (3.3).

If, for every $k \in \mathbb{N}$, there exist $\rho_{k}>r_{k}>0$ such that

$\left(F_{2}\right) a_{k}:=\inf _{\substack{u \in Z_{k} \\\|u\|=r_{k}}} \varphi(u) \rightarrow \infty, k \rightarrow \infty$,

$\left(F_{3}\right) b_{k}:=\max _{\substack{u \in Y_{k} \\\|u\|=\rho_{k}}} \varphi(u) \leq 0$,

$\left(F_{4}\right) \phi$ satisfies the nonsmooth $(\mathrm{PS})_{c}$ condition for every $c>0$, then $\phi$ has an unbounded sequence of critical values.

Proposition 3.8. (Nonsmooth dual Fountain theorem, see [37]) Assume $\left(F_{1}\right)$ is satisfied and there is a $k_{0}>0$ such that, for each $k \geq k_{0}$, there exists $\rho_{k}>\gamma_{k}>0$ such that

$$
\begin{aligned}
\left(D_{1}\right) a_{k}:=\inf _{\substack{u \in Z_{k} \\
\|u\|=\rho_{k}}} \varphi(u) \geq 0, \\
\left(D_{2}\right) b_{k}:=\max _{\substack{u \in Y_{k} \\
\|u\|=r_{k}}} \varphi(u)<0, \\
\left(D_{3}\right) d_{k}:=\inf _{\substack{u \in Z_{k} \\
\|u\| \leq \rho_{k}}} \varphi(u) \rightarrow 0, k \rightarrow \infty,
\end{aligned}
$$

$\left(D_{4}\right) \phi$ satisfies the nonsmooth (PS)* condition for every $c \in\left[d_{k_{0}}, 0\right)$, then $\phi$ has a sequence of negative critical values converging to 0 .

Remark 3.2. We say $\phi$ that satisfies the nonsmooth (PS)* condition at level $c \in \mathbb{R}$ (with respect to $\left(Y_{n}\right)$ ) if any sequence $\left\{u_{n}\right\} \subset X$ such that

$$
n_{j} \rightarrow \infty, u_{n_{j}} \in Y_{n_{j}}, \varphi\left(u_{n_{j}}\right) \rightarrow c,\left.m\right|_{Y_{n_{j}}}\left(u_{n}\right) \rightarrow 0
$$

contains a subsequence converging to a critical point of $\phi$. 


\section{Existence and multiplicity of solutions}

In this section, using the critical point theory, we give the existence and multiplicity results for problem (1.1). We shall use the following assumptions: $p^{-}$,

$$
\left(\mathbf{O}_{1}\right) \exists \delta_{1}>0, c_{3}>0 \text { and } q_{3} \in C(\bar{\Omega}) \text { with } q_{3}(x)<p_{a_{1}(x)}^{*}(x) \text { for } x \in \bar{\Omega} \text { and } q_{3}^{+}<\quad \text { such }
$$

that

$$
\begin{aligned}
& G_{1}(x, t) \geq c_{3} t^{q_{3}(x)}, \forall x \in \Omega, \forall t \in\left(0, \delta_{1}\right] . \\
& \left(\mathbf{O}_{2}\right) \exists \delta_{2}>0, c_{4}>0 \text { and } q_{4} \in C(\bar{\Omega}) \text { with } q_{4}(x)<p_{a_{2}(x)}^{*}(x) \text { for } x \in \bar{\Omega} \text { and } q_{4}^{-}>
\end{aligned}
$$

such

that

$$
\left|G_{2}(x, t)\right| \leq c_{4}|t|^{q_{4}(x)}, \forall x \in \Omega, \forall|t| \leq \delta_{2} .
$$

(S) For $i=1,2, G_{i}(x,-t)=G_{i}(x, t), \forall x \in \Omega, \forall t \in \mathbb{R}$.

\section{Remark 4.1.}

(1) It follows from $(\mathbf{A}),\left(\mathbf{A}_{2}\right)$ and $\left(\mathbf{O}_{2}\right)$ that

$$
\left|G_{2}(x, t)\right| \leq c_{4}|t|^{q_{4}(x)}+c_{5}|t|^{q_{2}(x)}, \forall x \in \Omega, \quad \forall t \in \mathbb{R} .
$$

(2)It follows from (A) and ( $\left.\mathbf{B}_{2}\right)$ that (see [33, p. 298])

$$
G(x, t) \geq c_{6}|t|^{1 / \theta}-c_{7}, \forall x \in \Omega, \forall t \in \mathbb{R} .
$$

The following is the main result of this article.

Theorem 4.1. Assume (P), (A), $\left(\mathbf{A}_{1}\right)$ hold.

(1) If $\left(\mathbf{B}_{1}\right)$ holds, then for every $\lambda \in \mathbb{R}$ and $\mu \leq 0$, problem (1.1) has a solution which is a minimizer of the corresponding functional $\phi$.

(2) If $\left(\mathbf{B}_{1}\right),\left(\mathbf{A}_{2}\right),\left(\mathbf{O}_{1}\right),\left(\mathbf{O}_{2}\right)$ hold, then for every $\lambda>0$ and $\mu \leq 0$, problem (1.1) has a nontrivial solution $v_{1}$ such that $v_{1}$ is a minimizer of $\phi$ and $\phi\left(v_{1}\right)<0$.

(3) If $\left(\mathbf{A}_{2}\right),\left(\mathbf{B}_{2}\right),\left(\mathbf{O}_{2}\right)$ hold, then for every $\mu>0$, there exists $\lambda_{0}(\mu)>0$ such that when $\lambda \mid \leq \lambda_{0}(\mu)$, problem (1.1) has a nontrivial solution $u_{1}$ such that $\phi\left(u_{1}\right)>0$.

(4) If $\left(\mathbf{A}_{2}\right),\left(\mathbf{B}_{2}\right),\left(\mathbf{O}_{1}\right),\left(\mathbf{O}_{2}\right)$ holds, then for every $\mu>0$, there exists $\lambda_{0}(\mu)>0$ such that when $0<\lambda \leq \lambda_{0}(\mu)$, problem (1.1) has two nontrivial solutions $u_{1}$ and $v_{1}$ such that $\phi\left(u_{1}\right)>0$ and $\phi\left(v_{1}\right)<0$.

(5) If $\left(\mathbf{A}_{2}\right),\left(\mathbf{B}_{2}\right),\left(\mathbf{O}_{1}\right),\left(\mathbf{O}_{2}\right)$ and (S) holds, then for every $\mu>0$ and $\lambda \in \mathbb{R}$, problem (1.1) has a sequence of solutions $\left\{ \pm u_{k}\right\}$ such that $\phi\left( \pm u_{k}\right) \rightarrow \infty$ as $k \rightarrow \infty$.

(6) If $\left(\mathbf{A}_{2}\right),\left(\mathbf{B}_{2}\right),\left(\mathbf{O}_{1}\right),\left(\mathbf{O}_{2}\right)$ and (S) holds, then for every $\lambda>0$ and $\mu \in \mathbb{R}$, problem (1.1) has a sequence of solutions $\left\{ \pm v_{k}\right\}$ such that $\phi\left( \pm v_{k}\right)<0$ and $\phi\left( \pm v_{k}\right) \rightarrow 0$ as $k \rightarrow \infty$.

Proof. We will use $c, c^{\prime}$ and $c_{i}$ as a generic positive constant. By Corollary 3.1, under the assumptions of Theorem 4.1, $\phi$ satisfies nonsmooth (PS) condition. We write

$$
\Psi_{1}(u)=\lambda \int_{\Omega} a_{1}(x) G_{1}(x, u) d x, \Psi_{2}(u)=\mu \int_{\Omega} a_{2}(x) G_{2}(x, u) d x,
$$

then $\Psi=\Psi_{1}+\Psi_{2}, \phi(u)=J(u)-\Psi(u)=J(u)-\Psi_{1}(u)-\Psi_{2}(u)$. Firstly, we use $\widehat{\Psi}_{i}$ to denote its extension to $L^{q_{i}(x)}(\Omega)$, where $i=1,2$. From (A) and Theorem 1.3.10 of [33] (or Chang [22]), we see that $\widehat{\Psi}_{i}(u)$ is locally Lipschitz on $L^{q_{i}(x)}(\Omega)$ and 
$\partial \widehat{\Psi}_{i}(u) \subseteq\left\{\xi_{i}(x) \in L^{q_{i}^{0}}(\Omega): \xi_{i}(u) \in \partial G_{i}(x, u)\right\}$ for a.e. $x \in \Omega$ and $i=1$, 2. In view of Proposition 2.4 and Theorem 2.2 of [22], we have that $\Psi_{i}=\left.\widehat{\Psi}_{i}\right|_{X}$ is also locally Lipschitz, and $\partial \Psi_{1}(u) \subseteq \lambda \int_{\Omega} a_{1}(x) \partial G_{1}(x, u) d x, \partial \Psi_{2}(u) \subseteq \mu \int_{\Omega} a_{2}(x) \partial G_{1}(x, u) d x$, (see [38]), where $\left.\widehat{\Psi}_{i}\right|_{X}$ stands for the restriction of $\widehat{\Psi}_{i}$ to $X$ for $i=1,2$. Therefore, $\phi$ is a locally Lipschitz functional on $\mathrm{X}$.

(1) Let $\lambda \in \mathbb{R}$ and $\mu \leq 0$. By (A),

$$
\left|\Psi_{1}(u)\right| \leq c_{1} \int_{\Omega} a_{1}(x)|u|^{q_{1}(x)} d x+c_{2} \leq c_{1}\left(|u|\left(q_{1}(x), a_{1}(x)\right)^{q_{1}^{+}}+c_{3} \leq c_{4}|| u||^{q_{1}^{+}}+c_{3} .\right.
$$

By $\left(\mathbf{B}_{1}\right), \Psi_{2}(u) \leq-\mu c_{0} \int_{\Omega} a_{2}(x) d x=c_{5}$. Hence $\varphi(u) \geq \frac{1}{p^{+}}\|u\|^{p^{-}}-c_{4}\|u\|^{q_{1}^{+}}-c_{6}$. By $\left(\mathbf{A}_{1}\right), q_{1}^{+}<p^{-}$, so $\phi$ is coercive, that is, $\phi(u) \rightarrow \infty$ as $\|u\| \rightarrow \infty$. Thus $\phi$ has a minimize which is a solution of (1.1).

(2) Let $\lambda>0, \mu \leq 0$ and the assumptions of (2) hold. By the above conclusion (1), $\phi$ has a minimize $v_{1}$. Take $v_{0} \in C_{0}^{\infty}(\Omega)$ such that $0 \leq v_{0}(x) \leq \min \left\{\delta_{1}, \delta_{2}\right\}$, $\int_{\Omega} a_{1}(x) v_{0}(x)^{q_{3}(x)} d x=d_{1}>0$ and $\int_{\Omega} a_{2}(x) v_{0}(x)^{q_{4}(x)} d x=d_{2}>0$. By $\left(\mathbf{O}_{1}\right)$ and $\left(\mathbf{O}_{2}\right)$ we have, for $t \in(0,1)$ small enough,

$$
\begin{aligned}
\varphi\left(t v_{0}\right) & =\int_{\Omega} \frac{1}{p(x)}\left|t \nabla v_{0}\right|^{p(x)} d x-\lambda \int_{\Omega} a_{1}(x) G_{1}\left(x, t v_{0}(x)\right) d x-\mu \int_{\Omega} a_{2}(x) G_{2}\left(x, t v_{0}(x)\right) d x \\
& \leq t^{p^{-}} \int_{\Omega} \frac{1}{p(x)}\left|\nabla v_{0}\right|^{p(x)} d x-\lambda \int_{\Omega} a_{1}(x) c_{3}\left(t v_{0}(x)\right)^{q_{3}(x)} d x \\
& -\mu \int_{\Omega} a_{2}(x) c_{4}\left(t v_{0}(x)\right)^{q_{4}(x)} d x \\
& \leq t^{p^{-}} \int_{\Omega} \frac{1}{p(x)}\left|\nabla v_{0}\right|^{p(x)} d x-t^{q_{3}^{+}} \lambda c_{3} d_{1}-t^{q_{4}^{-}} \mu c_{4} d_{2} .
\end{aligned}
$$

Since $q_{3}^{+}<p^{-}<q_{4}^{-}$, we can find $t_{0} \in(0,1)$ such that $\phi\left(t_{0} v_{0}\right)<0$, and this shows $\phi\left(v_{1}\right)=\inf _{u \in X} \phi(u)<0$. So $v_{1} \neq 0$ because $\phi(0)=0$. The conclusion (2) is proved.

(3) Let $\mu>0$ and the assumptions of (3) hold. By Remark 4.1.(1), for sufficiently small $\|u\|$

$$
\begin{aligned}
\Psi_{2}(u) & \leq \mu \int_{\Omega} a_{2}(x)\left(c_{4}|u|^{q_{4}(x)}+c_{5}|u|^{q_{2}(x)}\right) d x \\
& \leq \mu c_{4}\left(|u|_{\left(q_{4}(x), a_{2}(x)\right)}\right)^{q_{4}^{-}}+\mu c_{5}\left(|u|_{\left(q_{2}(x), a_{2}(x)\right)}\right)^{q_{2}^{-}} \\
& \leq \mu c_{8}\left(|| u||^{q_{4}^{-}}+|| u||^{q_{2}^{-}}\right) .
\end{aligned}
$$

Since $p^{+}<q_{2}^{-}$and $p^{+}<q_{4}^{-}$, there exists $\gamma>0$ and $\alpha>0$ such that $J(u)-\Psi_{2}(u) \geq \alpha$ for $u \in S_{\gamma}$. We can find $\lambda_{0}(\mu)>0$ such that when $|\lambda| \leq \lambda_{0}(\mu), \Psi_{1}(u) \leq \alpha / 2$ for $u \in S_{\gamma}$. So when $|\lambda| \leq \lambda_{0}(\mu), \phi(u) \geq \alpha / 2>0$ for $u \in S_{\gamma}$. By Remark 4.1.(2), noting that $1 / \theta>p^{+}>q_{1}^{+}$, we can find a $u_{0} \in X$ such that $\left\|u_{0}\right\|>\gamma$ and $\phi\left(u_{0}\right)<0$. By Proposition 3.6 problem (1.1) has a nontrivial solution $u_{1}$ such that $\phi\left(u_{1}\right)>0$.

(4) Let $\mu>0$ and the assumptions of (4) hold. By the conclusion (3), we know that, there exists $\lambda_{0}(\mu)>0$ such that when $0<\lambda \leq \lambda_{0}(\mu)$, problem (1.1) has a nontrivial 
solution $u_{1}$ such that $\phi\left(u_{1}\right)>0$. Let $\gamma$ and $\alpha$ be as in the proof of (3), that is, $\phi(u) \geq \alpha / 2$ $>0$ for $u \in S_{\gamma}$ By $\left(\mathbf{O}_{1}\right),\left(\mathbf{O}_{2}\right)$ and the proof of (2), there exists $w \in X$ such that $\|w\|<$ $\gamma$ and $\phi(w)<0$. It is clear that there is $v_{1} \in B_{\gamma}$, a minimizer of $\phi$ on $B_{\gamma}$. Thus $v_{1}$ is a nontrivial solution of $(1.1)$ and $\phi\left(v_{1}\right)<0$.

(5) Let $\mu>0, \lambda \in \mathbb{R}$ and the assumptions of (5) hold. By (S), we can use the nonsmooth version Fountain theorem with the antipodal action of $\mathbb{Z}_{2}$ to prove (5) (see Proposition 3.7). Denote

$$
\Psi(u)=\int_{\Omega} F(x, u) d x=\lambda \int_{\Omega} a \quad{ }_{1}(x) G_{1}(x, u) d x+\mu \int_{\Omega} a \quad{ }_{2}(x) G_{2}(x, u) d x .
$$

Let $\beta_{k}(\gamma)$ be as in Proposition 3.5. By Proposition 3.5, for each positive integer $n$, there exists a positive integer $k_{0}(n)$ such that $\beta_{k}(n) \leq 1$ for all $k \geq k_{0}(n)$. We may assume $k_{0}(n)<k_{0}(n+1)$ for each $n$. We define $\left\{\gamma_{k}: k=1,2, \ldots,\right\}$ by

$$
\gamma_{k}=\left\{\begin{array}{l}
n \text { if } k_{0}(n) \leq k<k_{0}(n+1) \\
1 \text { if } 1 \leq k<k_{0}(1)
\end{array}\right.
$$

Note that $\gamma_{k} \rightarrow \infty$ as $k \rightarrow \infty$. Then for $u \in Z_{k}$ with $\|u\|=\gamma_{k}$ we have

$$
\varphi(u)=\int_{\Omega} \frac{1}{p(x)}|\nabla u|^{p(x)} d x-\Psi(u) \geq \frac{1}{p^{+}}\left(\gamma_{k}\right)^{p^{-}}-1
$$

and consequently

$$
\inf _{u \in Z_{k},\|u\|=\gamma_{k}} \varphi(u) \rightarrow \infty \text { as } k \rightarrow \infty,
$$

i.e., the condition $\left(F_{2}\right)$ of Proposition 3.7 is satisfied.

By (A), $\left(\mathbf{A}_{1}\right),\left(\mathbf{B}_{2}\right)$ and Remark 4.1.(2), we have

$$
\varphi(u) \leq \frac{1}{p^{-}}|| u||^{p^{+}}+c_{1}|\lambda|\left(|u|_{\left(q_{1}(x), a_{1}(x)\right)}\right)^{q_{1}^{+}}-c_{6} \mu\left(|u|_{\left(1 / \theta, a_{2}(x)\right)}\right)^{1 / \theta}+c_{9} .
$$

Noting that $1 / \theta>p^{+}>q_{1}^{+}$and all norms on a finite dimensional vector space are equivalent each other, we can see that, for each $Y_{k}, \phi(u) \rightarrow-\infty$ as $u \in Y_{k}$ and $\|u\| \rightarrow$ $\infty$. Thus for each $k$ there exists $\rho_{k}>\gamma_{k}$ such that $\phi(u)<0$ for $u \in Y_{k} \cap S_{\rho k}$, so the condition $\left(F_{3}\right)$ of Proposition 3.7 is satisfied. As was noted earlier, $\phi$ satisfies nonsmooth (PS) condition. By Proposition 3.7 the conclusion (5) is true.

(6) Let $\lambda>0, \mu \in \mathbb{R}$ and the assumptions of (5) hold. Let us verify the conditions of the Nonsmooth dual Fountain theorem (see Proposition 3.8). By (S), $\phi$ is invariant on the antipodal action of $\mathbb{Z}_{2}$. For $\Psi(u)=\int_{\Omega} F(x, u) d x=\Psi_{1}(u)+\Psi_{2}(u)$ let $\beta_{k}(1)$ be as in Proposition 3.5, that is

$$
\beta_{k}(1)=\sup _{u \in Z_{k},\|u\| \leq 1}|\Psi(u)| .
$$

By Proposition 3.5, there exists a positive integer $k_{0}$ such that $\beta_{k}(1) \leq \frac{1}{2 p^{+}}$for all $k \geq$ $k_{0}$. Setting $\rho_{k}=1$, then for $k \geq k_{0}$ and $u \in Z_{k} \cap S_{1}$, we have

$$
\varphi(u) \geq \frac{1}{p^{+}}-\frac{1}{2 p^{+}}=\frac{1}{2 p^{+}}>0,
$$


which shows that the condition $\left(D_{1}\right)$ of Proposition 3.8 is satisfied.

Since $X=W_{0}^{1, p(x)}$ is the closure of $C_{0}^{\infty}(\Omega)$ in $W^{1, p(x)}(\Omega)$, we may choose $\left\{Y_{k}: k=1,2\right.$. $\ldots$,$\} , a sequence of finite dimensional vector subspaces of X$ defined by (3.5), such that $Y_{k} \subset C_{0}^{\infty}(\Omega)$ for all $k$. For each $Y_{k}$, because all norms on $Y_{k}$ are equivalent each other, there is $\varepsilon \in(0,1)$ such that for every $u \in Y_{k} \cap B_{\varepsilon},|u|_{\infty} \leq \min \left\{\delta_{1}, \delta_{2}\right\},|u|_{\left(q_{3}(x), a_{1}(x)\right)} \leq 1$ and $|u|_{\left(q_{4}(x), a_{2}(x)\right)} \leq 1$ By $\left(\mathbf{O}_{1}\right)$ and $\left(\mathbf{O}_{2}\right)$, for $u \in Y_{k} \cap B_{\varepsilon}$ we have

$$
\begin{aligned}
\varphi(u) & \leq \frac{1}{p^{-}}\|u\|^{p^{-}}-\lambda c_{3} \int_{\Omega} a_{1}(x)|u|^{q_{3}(x)} d x+|\mu| c_{4} \int_{\Omega} a_{2}(x)|u|^{q_{4}(x)} d x \\
& \leq \frac{1}{p^{-}}\|u\|^{p^{-}}-\lambda c_{3}\left(|u|_{\left(q_{3}(x), a_{1}(x)\right)}\right)^{q_{3}^{+}}+|\mu| c_{4}\left(|u|_{\left(q_{4}(x), a_{2}(x)\right)}\right)^{q_{4}^{-}} .
\end{aligned}
$$

Because $q_{3}^{+}<p^{-}<q_{4}^{-}$there exists $\gamma_{k} \in(0, \varepsilon)$ such that

$$
b_{k}:=\max _{u \in Y_{k},\|u\|=\gamma_{k}} \varphi(u)<0,
$$

thus the condition $\left(D_{2}\right)$ of Proposition 3.8 is satisfied.

Because $Y_{k} \cap Z_{k} \neq \varnothing$ and $\gamma_{k}<\rho_{k}$, we have

$$
d_{k}:=\inf _{u \in Z_{k},\|u\| \leq \rho_{k}} \varphi(u) \leq b_{k}:=\max _{u \in Y_{k},\|u\|=r_{k}} \varphi(u)<0 .
$$

On the other hand, for any $u \in Z_{k}$ with $\|u\| \leq 1=\rho_{k}$, we have $\phi(u)=J(u)-\Psi(u) \geq$ $-\Psi(u) \geq-\beta_{k}(1)$. Noting that $\beta_{k} \rightarrow 0$ as $k \rightarrow \infty$, we obtain $d_{k} \rightarrow 0$, i.e., $\left(D_{3}\right)$ of Proposition 3.8 is satisfied.

Finally let us prove that $\phi$ satisfies nonsmooth (PS) ${ }_{c}^{*}$ condition for every $c \in R$. Suppose $\left\{u_{n_{j}}\right\} \subset X, n_{j} \rightarrow \infty, u_{n_{j}} \in Y_{n_{j}}, \varphi\left(u_{n_{j}}\right) \rightarrow c$ and $\left.m\right|_{Y_{n_{j}}}\left(u_{n_{j}}\right) \rightarrow 0$. Similar to the process of verifying the (PS) condition in the proof of Proposition 3.3, we can get $u_{n_{j}} \rightarrow u$ in $X$. Let us prove $0 \in \partial \phi(u)$ below. Notice that

$$
0 \leq m(u)=m(u)-m\left(u_{n_{j}}\right)+m\left(u_{n_{j}}\right)=m(u)-m\left(u_{n_{j}}\right)+\left.m\right|_{Y_{n_{j}}}\left(u_{n_{j}}\right) .
$$

Using Proposition 2.8.4, Going to limit in the right side of above equation, we have

$$
m(u) \leq 0,
$$

so $m(u) \equiv 0$, i.e., $0 \in \partial \phi(u)$, this shows that $\phi$ satisfies the nonsmooth (PS) $)_{c}^{*}$ condition for every $c \in \mathbb{R}$. So all conditions of Proposition 3.8 are satisfied and the conclusion (6) follows from Proposition 3.8. The proof of Theorem 4.1 is complete.

\section{Remark 4.2}

Theorem 4.1 includes several important special cases. In particular, in the case of the problem (1.4), i.e., in the case that

$$
a_{1}(x)=\frac{1}{|x|^{s_{1}(x)}}, a_{2}(x)=\frac{1}{|x|^{s_{2}(x)}},
$$

all conditions of Theorem 4.1 are satisfied provided $(\mathbf{P}),\left(\mathbf{A}^{*}\right),\left(\mathbf{A}_{1}\right)$, and $\left(\mathbf{A}_{2}\right)$ hold. 


\section{Acknowledgements}

The authors are very grateful to the anonymous referees for their valuable suggestions. Research supported by the NSFC (Nos. 11061030, 10971087), 1107RJZA223 and the Fundamental Research Funds for the Gansu Universities.

\section{Authors' contributions}

GD conceived of the study, and participated in its design and coordination and helped to draft the manuscript. RM participated in the design of the study. All authors read and approved the final manuscript.

\section{Competing interests}

The authors declare that they have no competing interests.

Received: 5 November 2011 Accepted: 9 February 2012 Published: 9 February 2012

\section{References}

1. Rüžzička, M: Electro-rheological Fluids: Modeling and Mathematical Theory. Springer, Berlin (2000)

2. Zhikov, W: Averaging of functionals of the calculus of variations and elasticity theory. Math USSR IZV. 9, 33-66 (1987)

3. Antontsev, SN, Shmarev, SI: A model porous medium equation with variable exponent of nonlinearity: existence, uniqueness and localization properties of solutions. Nonlinear Anal. 60, 515-545 (2005)

4. Antontsev, SN, Rodrigues, JF: On stationary thermo-rheological viscous flows. Ann Univ Ferrara Sez Sci Mat. 52, 19-36 (2006). doi:10.1007/s11565-006-0002-9

5. Chen, Y, Levine, S, Rao, M: Variable exponent, linear growth functionals in image restoration. SIAM J Appl Math. 66(4), 1383-1406 (2006). doi:10.1137/050624522

6. Diening, L, Hästö, P, Nekvinda, A: Open problems in variable exponent Lebesgue and Sobolev spaces. Drábek, P, Rákosník, J, FSDONA04 Proceedings, 38-58, Milovy, Czech Republic. (2004)

7. Fan, XL, Zhao, D: On the Spaces $L^{L^{(x)}}$ and $W^{m, P(x)}$. J Math Anal Appl. 263, 424-446 (2001). doi:10.1006/jmaa.2000.7617

8. Harjulehto, P, Hästö, P: An overview of variable exponent Lebesgue and Sobolev spaces. In: Herron D (ed.) Future Trends in Geometric Function Theory. pp. 85-93. RNC Workshop, Jyväskylä (2003)

9. Samko, S: On a progress in the theory of Lebesgue spaces with variable exponent maximal and singular operators. Integr Trans Spec Funct. 16, 461-482 (2005). doi:10.1080/10652460412331320322

10. Jikov, W, Kozlov, SM, Oleinik, OA: Homogenization of Differential Operators and Integral Functionals (Translated from the Russian by Yosifian, GA). Springer, Berlin (1994)

11. Zhikov, W: On some variational problems. Russ J Math Phys. 5, 105-116 (1997)

12. Dai, G: Three symmetric solutions for a differential inclusion system involving the $(p(x), q(x))$-Laplacian in $\bigotimes^{N}$. Nonlinear Anal. 71, 1763-1771 (2009). doi:10.1016/j.na.2009.01.012

13. Dai, G: Infinitely many solutions for a Neumann-type differential inclusion problem involving the $p(x)$-Laplacian. Nonlinear Anal. 70, 2297-2305 (2009). doi:10.1016/j.na.2008.03.009

14. Dai, G: Infinitely many solutions for a hemivariational inequality involving the $p(x)$-Laplacian. Nonlinear Anal. 71 186-195 (2009). doi:10.1016/j.na.2008.10.039

15. Dai, G: Three solutions for a Neumann-type differential inclusion problem involving the $p(x)$-Laplacian. Nonlinear Anal. 70, 3755-3760 (2009). doi:10.1016/..na.2008.07.031

16. Dai, G: Infinitely many solutions for a differential inclusion problem in $\bigotimes^{N}$ involving the $p(x)$-Laplacian. Nonlinear Anal. 71, 1116-1123 (2009). doi:10.1016/j.na.2008.11.024

17. Fan, XL, Han, XY: Existence and multiplicity of solutions for $p(x)$-Laplacian equations in $R^{N}$. Nonlinear Anal. 59, 173-188 (2004)

18. Fan, XL: On the sub-supersolution methods for $p(x)$-Laplacian equations. J Math Anal Appl. 330, 665-682 (2007). doi:10.1016/j.jmaa.2006.07.093

19. Fan, XL, Zhang, QH: Existence of solutions for $p(x)$-Laplacian Dirichlet problems. Nonlinear Anal. 52, 1843-1852 (2003). doi:10.1016/50362-546X(02)00150-5

20. Fan, XL, Zhang, QH, Zhao, D: Eigenvalues of $p(x)$-Laplacian Dirichlet problem. J Math Anal Appl. 302, 306-317 (2005). doi:10.1016/j.jmaa.2003.11.020

21. Fan, XL: Solutions for $p(x)$-Laplacian Dirichlet problems with singular coefficients. J Math Anal Appl. 312, 464-477 (2005). doi:10.1016/j.jmaa.2005.03.057

22. Chang, KC: Variational methods for nondifferentiable functionals and their applications to partial differential equations. J Math Anal Appl. 80, 102-129 (1981). doi:10.1016/0022-247X(81)90095-0

23. Kourogenis, NC, Papageorgiou, NS: Nonsmooth crical point theory and nonlinear elliptic equation at resonance. KODAl Math J. 23, 108-135 (2000). doi:10.2996/kmj/1138044160

24. Ricceri, B: A general variational principle and some of its applications. J Comput Appl Math. 113, 401-410 (2000). doi:10.1016/50377-0427(99)00269-1

25. Ricceri, B: On a three critical points theorem. Arch Math (Basel). 75, 220-226 (2000). doi:10.1007/s000130050496

26. Marano, S, Motreanu, D: Infinitely many critical points of non-differentiable functions and applications to a Neumanntype problem involving the p-Laplacian. J Diff Equ. 182, 108-120 (2002). doi:10.1006/jdeq.2001.4092

27. Marano, SA, Motreanu, D: On a three critical points theorem for non differentiable functions and applications to nonlinear boundary value problems. Nonlinear Anal. 48, 37-52 (2002). doi:10.1016/S0362-546X(00)00171-1

28. Diening, L: Riesz potential and Sobolev embeddings on generalized Lebesque and Sobolev Spaces $L^{p(\cdot)}$ and $W^{k, p}(\cdot)$ Math. Nachr. 268, 31-43 (2004). doi:10.1002/mana.200310157

29. Fan, XL, Shen, JS, Zhao, D: Sobolev embedding theorems for spaces $W^{k, p(x)}(\Omega)$. J Math Anal Appl. 262, 749-760 (2001). doi:10.1006/jmaa.2001.7618

30. Samko, SG: Hardy-Littlewood-Stein-Weiss inequality in the Lebesgue spaces with variable exponent. Fract Calc Appl Anal. 6(4), 421-440 (2003)

31. Kovacik, O, Rakosnik, J: On spaces $L^{p(x)}(\Omega)$ and $W^{k, p(x)}(\Omega)$. Czechoslovak Math J. 41, 592-618 (1991)

32. Clarke, FH: Optimization and Nonsmooth Analysis. Wiley, New York (1983) 
33. Gasiéski, L, Papageorgiou, NS: Nonsmooth Critical Point Theory and Nonlinear Boundary Value Problems. Chapman and Hall/CRC, Boca Raton (2005)

34. Zhao, JF: Structure Theory of Banach Spaces (in Chinese). Wuhan University Press, Wuhan (1991)

35. Garcia Azorero, JP, Peral Alonso, I: Hardy inequalities and some critical elliptic and parabolic problems. J Diff Equ. 144, 441-476 (1998). doi:10.1006/jdeq.1997.3375

36. Dai, G: Nonsmooth version of Fountain theorem and its application to a Dirichlet-type differential inclusion problem. Nonlinear Anal. 72, 1454-1461 (2010). doi:10.1016/.na.2009.08.029

37. Dai, G, Wang, WT, Feng, LL: Nonsmooth version of dual Fountain theorem and its application to a differential inclusion problem. Acta Math Sci Ser A Chin Ed 32(2012).1, 18-28

38. Kristály, A: Infinitely many solutions for a differential inclusion problem in $\bigotimes^{N}$. J Diff Equ. 220, 511-530 (2006). doi:10.1016/j.jde.2005.02.007

doi:10.1186/1687-2770-2012-11

Cite this article as: Dai et al:: Existence of solutions for a differential inclusion problem with singular coefficients

involving the $p(x)$-Laplacian. Boundary Value Problems 2012 2012:11.

\section{Submit your manuscript to a SpringerOpen ${ }^{\circ}$} journal and benefit from:

- Convenient online submission

- Rigorous peer review

- Immediate publication on acceptance

- Open access: articles freely available online

- High visibility within the field

- Retaining the copyright to your article

Submit your next manuscript at $\gg$ springeropen.com 